\title{
Approximate Analytic Solution of a Self-Similar Piston Moving in an Inhomogeneous Medium
}

By B. N. Prasad

Kolhan University

Abstract- Self-similar motion for the flow between a piston and strong shock propagating in a non uniform ideal gas at rest has been studied. The solution to the problem is similar to that of hypersonic flows past the power law bodies. The gas ahead of the shock is assumed to be uniform and at rest. This is considered as a particular case of radiative piston problem. The shock is assumed to be very strong and propagating in a medium at rest in which density obeys power laws. This problem with spherical symmetry has got importance in astrophysics. To solve the gas dynamics problem, Chernyii's expansion techniques have been used in which flow variables are expanded in a series of powers of $\varepsilon$, the density ratio across the strong shock. The approximate analytic solution has been obtained in closed form to the zeroth approximation. The problem discussed belongs to the self-similar motion of the first kind. The resulting analytic solution gives the flow variables distribution for plane, cylindrical, and spherical symmetry for different cases which satisfy the similarity conditions with accurate trend and values.

Keywords: self-similar motion, similarity solution, chernyii's technique, piston problem, strong shock, plane-cylindrical \& spherical symmetry, radiative gas dynamics.

GJSFR-A Classification: FOR Code: 240101

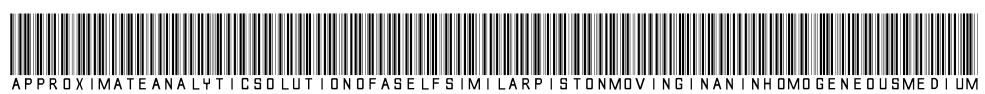

Strictly as per the compliance and regulations of:

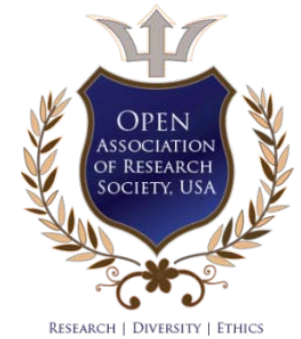

(C) 2020. B. N. Prasad. This is a research/review paper, distributed under the terms of the Creative Commons AttributionNoncommercial 3.0 Unported License http://creativecommons.org/licenses/by-nc/3.0/), permitting all non commercial use, distribution, and reproduction in any medium, provided the original work is properly cited. 


\title{
Approximate Analytic Solution of a Self-Similar Piston Moving in an Inhomogeneous Medium
}

\author{
B. N. Prasad
}

\begin{abstract}
Self-similar motion for the flow between a piston and strong shock propagating in a non uniform ideal gas at rest has been studied. The solution to the problem is similar to that of hypersonic flows past the power law bodies. The gas ahead of the shock is assumed to be uniform and at rest. This is considered as a particular case of radiative piston problem. The shock is assumed to be very strong and propagating in a medium at rest in which density obeys power laws. This problem with spherical symmetry has got importance in astrophysics. To solve the gas dynamics problem, Chernyii's expansion techniques have been used in which flow variables are expanded in a series of powers of $\varepsilon$, the density ratio across the strong shock. The approximate analytic solution has been obtained in closed form to the zeroth approximation. The problem discussed belongs to the self-similar motion of the first kind. The resulting analytic solution gives the flow variables distribution for plane, cylindrical, and spherical symmetry for different cases which satisfy the similarity conditions with accurate trend and values.
\end{abstract}

Keywords: self-similar motion, similarity solution, chernyii's technique, piston problem, strong shock, plane-cylindrical \& spherical symmetry, radiative gas dynamics.

Subject Classification:

1. AMS(American Mathematical Society) -- 76L05 (Shockwaves \& Blast Waves)

2. PACS(Phys. \& Astron. Classification Scheme) AIP (American Inst. of Phys.) -- 47.40(Compressible Flow, Shock \& Detonation Phenomena)

\section{INTRODUCTION}

T he propagation of a blast wave in an inhomogeneous medium, with ambient density given by $\rho_{o} \alpha x^{-\alpha}$, where $x$ is the distance from the center of explosion and $\alpha$ is a positive number, has been analytically studied by Sedov ${ }^{[1]}$ and Rogers ${ }^{[2]}$. The energy of the explosion does not vary with time, and the problem is self-similar. The uniform expansion of a piston in a homogeneous medium, taking into account the counterpressure across the shock, was numerically solved by Taylor ${ }^{[3]}$. Bhatnagar and $\mathrm{Lal}^{\left[{ }^{[4]}\right.}$ have also considered explosion problem for finite shock strength in a non-homogeneous medium, both when the selfgravitation of the gas is important, and when it is negligible. The motion produced by a piston moving according to the general law $\mathrm{R}=\mathrm{C} \mathrm{t}^{\mathrm{n}+1}$, where $\mathrm{n}>-1$, for strong shocks, has been considered by Kochina and

Author: Department of Mathematics, Kolhan University, Chaibasa833201(India). e-mail: bnprasad60@hotmail.com
Melnikov ${ }^{[5]}$ and Rogers $^{[6]}$, besides others, by solving numerically the ordinary differential equations obtained through similarity transformations. Wang ${ }^{[7]}$ has obtained an approximate solution of a plane radiative piston. Sachdev and Ashra ${ }^{[8]}$ have considered the problems of plane, spherical, and, cylindrical pistons moving in an inhomogeneous medium and have obtained solutions in terms of Incomplete Beta functions. The problem of Sedov $^{[1]}$ and Taylor ${ }^{[3]}$ was extended by Krashaninikova ${ }^{[9]}$ to the case when the piston expands non-uniformly with a velocity $U$ is given by

$$
U=U_{0} t^{n},(n>-1) \ldots
$$

where $U_{0}$ is a constant.

In this paper, self-similar solutions for the flow between a piston and the strong shock propagating non-uniformly in an ideal gas at rest obeying the powerlaw density distribution are investigated. Ranga Rao and Purohit ${ }^{[10]}$ have studied this problem numerically. The solutions of this problem are similar to that of the solutions of hypersonic flow past the power-law bodies obtained by Lees and Kubota ${ }^{[11]}$. They have shown that the condition for the existence of the solution is$\frac{v}{v+2}<\mathrm{n} \leq 0$,

where $v=1,2,3$ for plane, cylindrical and spherical flows respectively. In all these works, the gas ahead of the shock is assumed to be uniform and at rest. Helliwell ${ }^{[12]}$ studied the piston problem, in which the piston velocity is assumed to be of the form (1.1) and the density of gas ahead of the shock satisfies the law

$$
\rho_{\mathrm{o}}=\operatorname{Ar}^{-\mathrm{w}}(\mathrm{w}>0) \text {. }
$$

where $\mathrm{A}$ is a constant.

This problem has been considered as a particular case of a radiative piston problem in which there exists, by dimensional considerations, a relation between $n$ and $w$, namely

$n=-w / w+5$.

In this paper, we consider the self-similar piston problem in which the piston velocity obeys the law (1.1). The shock is assumed to be strong and propagating in a medium at rest in which the density obeys equation (1.2). The problem with spherical symmetry has got importance in astrophysics, Parker ${ }^{[13]}$. We first observe that there are only independent dimensional constants $U_{0}$ and $A$ involved in the problem, and hence selfsimilarity exists. Also, there cannot be, in general, any explicit relation between $n$ and $w$ as given by Helliwel[ ${ }^{[12]}$. 
On the other hand, it is shown that for all physically meaningful flows the ranges for $\mathrm{n}$ and $\mathrm{w}$ are

$$
\frac{-(v-w)}{v+2-w}<\mathrm{n}<\frac{-w(\gamma-1)}{[w(\gamma-1)+2]} \quad \text { and } 0<\mathrm{W}<\frac{v}{\gamma}
$$

where $\gamma$ is the ratio of specific heats. These conditions on $\mathrm{n}$ ensure the finiteness of density and pressure drag on the piston surface. Also, it is shown that when $\mathrm{n}$ $=\frac{w(\gamma-1)}{[w(\gamma-1)+2]}$,

the flow becomes homentropic. The problem with $\gamma=\frac{7}{5}$, $\mathrm{n}=-\mathrm{w} / \mathrm{w}+5$ considered by Helliwell ${ }^{[12]}$ corresponds to homentropic flow. Numerical solutions for $v=3, \gamma=\frac{7}{5}$, and $w=1.5$ are given using the Adam Moulton method.

Chernyii $^{[14]}$ has given an expansion technique to solve the gas-dynamics problem. The expansion parameter $\varepsilon=\frac{\gamma-1}{\gamma+1}$, which occurs in the shock boundary conditions where $\gamma$ is the density ratio across a strong shock. For gases when $\gamma$ generally varies between 1 and $5 / 3$, this parameter is sufficiently small. He has considered the motion of a piston and the big explosion problem in a uniform medium, restricting mostly to zeroth-order solution. In an earlier paper, Chernyii ${ }^{[15]}$ has given a particular solution of the piston problem in a homogeneous medium in an integral form to first order in $\varepsilon$. Wang ${ }^{[]]}$has also obtained an approximate analytic solution of a plane radiating piston, using Chernyii's ${ }^{[15]}$ technique. In this paper, we have employed Chernyii's ${ }^{[15]}$ technique, in which flow variables are expanded in power series in $\varepsilon$ and have obtained approximate analytic solutions in closed form to the zeroth approximation. This problem belongs to the class of self-similar motion of the first kind in which the similarity exponent occurring in the law of shock propagation can be determined in advance from physical considerations.

\section{il. Basic EQUations}

The partial differential equations of motion, continuity and energy for one-dimensional unsteady flow of a perfect gas are transformed into a set of ordinary differential equations,

$$
\begin{gathered}
\lambda(v-\delta) v^{\prime}+\frac{\mathrm{P}^{\prime}}{\mathrm{R}}+v(v-1)-(\mathrm{W}-2) \frac{\mathrm{P}}{\mathrm{R}}=0 \\
\lambda\left[v^{\prime}+(v-\delta) \frac{\mathrm{R}^{\prime}}{\mathrm{R}}\right]+(v-\mathrm{w}) v=0 \\
\lambda(v-\delta)\left[\frac{\mathrm{P}^{\prime}}{\mathrm{P}} \gamma \frac{\mathrm{R}^{\prime}}{\mathrm{R}}\right]-2+[\mathrm{W}(\gamma-1)+2] v=0
\end{gathered}
$$

by the following transformations

$$
\begin{gathered}
v=\frac{r}{\mathrm{t}} \mathrm{V}(\lambda), \quad \mathrm{R}=\frac{\mathrm{A}}{\mathrm{r}^{\mathrm{w}}} \mathrm{R}(\lambda), \quad \mathrm{p}=\frac{\mathrm{A}}{\mathrm{r}^{\mathrm{w}-2} \mathrm{t}^{2}} \mathrm{P}(\lambda) \\
\lambda=\left(\frac{\delta \bar{\lambda}}{\mathrm{U}_{0}}\right) \mathrm{rt}^{-\delta}, \quad \delta=1+\mathrm{n}
\end{gathered}
$$

The similarity variable $\lambda$ is took in the form (2.5) by considering $U_{0}$ and $A$ as the basic dimensional constants involved in the problem and, it takes values $\bar{\lambda}$ and 1 at the piston surface and behind the shock respectively. $V, R$, and $P$ are non-dimensional reduced particle velocity, density and, pressure, respectively.

The boundary conditions for the strong shock are

$$
\mathrm{V}(1)=\frac{2 \delta}{\gamma+1}, \quad \mathrm{R}(1)=\frac{\gamma+1}{\gamma-1}, \mathrm{P}(1)=\frac{2 \delta^{2}}{\gamma+1}
$$

and the kinematic condition on the piston gives

$$
v(\bar{\lambda})=\delta
$$

For the existence of solutions in the self-similar form, certain similarity conditions are to be satisfied. The total energy of the flow between the piston surface and the shock front, using (2.4) and (2.5), can be written as

$E=A K_{v}\left(\frac{U_{0}}{\delta \bar{\lambda}}\right)^{v+2-\mathrm{w}_{\mathrm{t}}} . \delta(v+2-\mathrm{w})-2 \int_{\lambda}^{1}\left(\frac{1}{2} \mathrm{RV}^{2}+\frac{\mathrm{p}}{\gamma-1}\right) \lambda^{v+1-\mathrm{w}} \mathrm{d} \lambda$

where

$$
K_{v}=2^{v-1} \pi \frac{(v-1)(4-v)}{2} .
$$

For the flows driven out by the piston, the energy always increases with time. This is possible only if

$$
n>-\left(\frac{v-\mathrm{w}}{v+2-\mathrm{w}}\right), \mathrm{w}<\mathrm{v} \ldots \ldots . \ldots
$$

The second condition of (2.5) is required to make sure that for all physically meaningful solutions, $\delta$ lies between zero and one. These conditions ensure that the pressure drag on the piston is finite. Further, it is necessary that $\frac{d v}{d \lambda}<0$ in the domain of interest. Thus, it follows that the density at the piston surface is finite if

$$
\frac{2}{v y+2-w}<\delta \leq \frac{2}{w(\gamma-1)+2} \ldots \ldots \ldots \ldots
$$

For all gases (with $\gamma>1$ ) the ranges for $\mathrm{n}$ and $\mathrm{w}$ can be obtained from (2.10) and (2.11) as

$$
-\frac{(v-w)}{v+2-w}<n \leq-\frac{w(\gamma-1)}{w(\gamma-1)+2}, 0<w<\frac{v}{\gamma} \ldots \ldots
$$

These are the conditions on $n$ and $w$ for the existence of physically meaningful solutions.

Following Chernyii's ${ }^{[15]}$ technique, we expand reduced flow variables as

$$
\begin{aligned}
& V=V^{(0)}+\varepsilon V^{(1)}+\varepsilon^{2} V^{(2)}+\cdots . \\
& R=\frac{R^{(0)}}{\varepsilon}+R^{(1)}+\varepsilon R^{(2)}+\cdots \ldots \ldots \\
& P=P^{(0)}+\varepsilon P^{(1)}+\varepsilon^{2} P^{(2)}+\ldots \ldots \ldots
\end{aligned}
$$

We substitute expansions (2.13) to (2.15) into equations (2.1) to (2.3) and obtain the following set of differential equations for the zeroth approximation-

$$
\begin{gathered}
\lambda\left[\mathrm{R}^{(0)} \mathrm{V}^{(0)} \frac{\mathrm{dV}(0)}{\mathrm{d} \lambda}-\delta \mathrm{R}^{(0)} \frac{\mathrm{dV}(0)}{\mathrm{d} \lambda}\right]+\mathrm{R}^{(0)} \mathrm{V}^{2(0)}-\mathrm{R}^{(0)} \mathrm{V}^{(0)}=0 \ldots \\
\lambda\left[\mathrm{R}^{(0)} \frac{\mathrm{d} \mathrm{V}^{(0)}}{\mathrm{d} \lambda}+\mathrm{V}^{(0)} \frac{\mathrm{dR} \mathrm{R}^{(0)}}{\mathrm{d} \lambda}-\delta \frac{\mathrm{dR}(0)}{\mathrm{d} \lambda}\right]+(v-\mathrm{w}) \mathrm{R}^{(0)} \mathrm{V}^{(0)}=0 \ldots \\
\lambda \mathrm{V}^{(0)}\left[\mathrm{R}^{(0)} \frac{\mathrm{dP}(0)}{\mathrm{d} \lambda}-\gamma \mathrm{P}^{(0)} \frac{\mathrm{dR}^{(0)}}{\mathrm{d} \lambda}\right]-\delta \lambda\left[\mathrm{R}^{(0)} \frac{\mathrm{dP}^{(0)}}{\mathrm{d} \lambda}-\gamma \mathrm{P}^{(0)} \frac{\mathrm{dR}^{(0)}}{\mathrm{d} \lambda}\right]
\end{gathered}
$$




$$
-2 P^{(0)} R^{(0)}+[w(\gamma-1)+2] R^{(0)} V^{(0)} P^{(0)}=0 \ldots
$$

The differential equations for the first and second order approximations were too complicated to be amenable to analytic solutions.

The boundary conditions at the shock become

$$
V^{(0)}=\frac{2 \delta}{\gamma+1}, R^{(0)}=1, P^{(0)}=\frac{2 \delta^{2}}{v+1} \ldots \ldots \ldots
$$

Integrating equation (2.16) to (2.18) with boundary conditions (2.19), we obtain the solution as

$$
\begin{aligned}
& V=V^{(0)}=\frac{1-\sqrt{1-4\left(\frac{1-\delta}{\delta}\right) A \lambda^{1 / \delta}}}{2\left(\frac{1-\delta}{\delta}\right)} \ldots \ldots \ldots \\
& R=R^{(0)}=\frac{B}{v-\delta} e^{\omega} \ldots \ldots \ldots \ldots \ldots \ldots \ldots \\
& P=P^{(0)}=C R^{\gamma} e^{-\epsilon} \lambda^{-2 / \delta} \ldots \ldots \ldots \ldots
\end{aligned}
$$

where, $A=\frac{2 \delta(\gamma+2 \delta)}{(\gamma+1)^{2}}$

$$
\begin{aligned}
& \omega=-(v-\mathrm{w})\left\{A \lambda^{1 / \delta}+\frac{S A^{2} \lambda^{2 / \delta}}{2}-\left(A+\frac{S A^{2}}{2}\right)\right. \\
& \left.+\frac{1}{\delta}\left(A^{2} \frac{\lambda^{2 / \delta}}{2}+S^{2} A^{4} \lambda^{4 / \delta}+2 S A^{3} \frac{\lambda^{3 / \delta}}{3}\right)\right\} \\
& -\frac{1}{\delta}\left(\frac{A^{2}}{2}+\frac{\delta^{2} A^{4}}{4}+2 S \frac{\lambda^{3}}{3}\right) \\
& S=\frac{1-\delta}{\delta}, \quad B=\left[R(\mathrm{v}-\delta) e^{-\omega}\right]_{\lambda=1} \\
& C=\left[P R^{-\gamma} \lambda^{2 / \delta} e^{\omega}\right]_{\lambda=1} \\
& \epsilon=-\left\{\eta A+\frac{A^{2}}{2}\left(\eta S+\frac{\eta \delta-2}{\delta^{2}}\right)+\left(\frac{\eta \delta-2}{\delta^{2}}\right)\left(\frac{A^{4} S^{2}}{4}+\frac{2 S A^{3}}{3}\right)\right\}+ \\
& \eta A \lambda^{1 / \lambda}-\frac{\lambda^{2 / \delta} A^{2}}{2}\left(\eta S+\frac{\eta \delta-2}{\delta^{2}}\right)+\frac{\eta \delta-2}{\delta^{2}}\left(\frac{S^{2} K^{4} \lambda^{4 / \delta}}{4}+\frac{2 S A^{3} \lambda^{3 / \delta}}{3}\right) \\
& \eta=w(\gamma-1)+2
\end{aligned}
$$

\section{Results AND Discussions}

Equations (2.20) to (2.21) give the solutions for the reduced particle velocity, density, and pressure up to zeroth approximation. The differential equations for the first and second-order terms in $\varepsilon$ were not amenable to an analytic solution. The error in the solution is of order $\mathrm{O}(\gamma)$, which is small if $\gamma$ is of $\mathrm{O}(1)$. Usually, the solution is obtained by integrating similarity equations (2.1) to (2.3) starting with the known values of flow variables at the shock, given by equation (2.6) and imposing the condition that the solution curve must pass through the appropriate singular point. Ranga Rao and Purohit ${ }^{[10]}$ have given numerical solutions for the spherical piston for $\gamma=\frac{7}{5}, \mathrm{w}=1.5$, by integrating the ordinary differential equation in terms of a new dependent variable, $\mathrm{Z}=\gamma \quad \frac{P}{\mathrm{R}}$ and $\mathrm{V}$. They started the integration from the known value at the shock and continuing until the value $\bar{\lambda}$ is reached such that $V(\bar{\lambda})=\delta$. Our analytic solutions give the flow variables distributions for plane, cylindrical and spherical symmetry for different values of $\mathrm{n}$ and $\mathrm{w}$, which satisfy the similarity conditions (2.10) and (2.12) with accurate trends and values.

Solutions for the flow between the piston and shock have been depicted graphically in figures1 to 6, for the spherical, cylindrical and plane symmetry for different values of $n$, when $\gamma=\frac{7}{5}$, which is of importance in astrophysics.

\section{References Références Referencias}

1. L. I. Sedov, On some steady motions of a compressible fluid, P.M.M. 9, 293, 1945.

2. M. H. Rogers, Analytic solutions for the blast wave problem with an atmosphere of varying density, Astrophysics J., 125, 478, 1957.

3. G. Taylor, The formation of a blast wave by a very intense explosion, Proc. R. Soc., A, 201, 159, 1950.

4. P. L. Bhatnagar and Purshotam Lal, Propagation of a spherical shock in aninhomogeneous selfgravitating or non-gravitating system, II Nuovo Cimento, 40, 483, 1965.

5. N. N. Kochina and N.s. Melnikova, On the unsteady motion of the gas driven outwards by a piston, neglecting the counter pressure, Prikl. Mat. Mekh, 22, 622, 1958.

6. M. H. Rogers, The propagation and structure of shock waves of varying strength in a self-gravitating gas sphere, Proc. R. Soc, A, 235, 120, 1956.

7. K. C. Wang, Approximate Solution of a plane radiating piston problem, Phys. Fluids, 9, 1922.

8. P. L. Sachdev and S. Ashraf, Approximate analytical solution of spherical, cylindrical and plane piston problems in an inhomogeneous medium, Indian Journal of Pure and Applied Maths., 1, 579, 1970.

9. N. L. Krashaninikova, On the unsteady motion of a gas displaced by a piston, Bull. Acad. Si., V.R.S.S. OTN8, 1955.

10. M. P. Rangaroa and S.C. Purohit, A self-similar piston problem, Jr. of Engg. Maths., 5, 257, 1971.

11. L. Lee and T. Kubota, Inviscid Hypersonic flow over blunt nosed slender bodies, J.Aero. Sci., 24, 195, 1957.

12. J. B. Helliwell, Self-similar piston problem with radiative heat transfer, J. Fluid Mech. 37, 497, 1969. 
13. E. N. Parker, Interplanetry dynamical processes, Inter Science Publications, 1963.

14. G. G. Chernyii, Application of integral relationships in problems of propagation of strong shock waves, Prikl. Mat. Mekh. 24, 159, 1960.
15. G. G. Chernyii, Introduction to Hypersonic Flow, Academic Press Inc., New York, 1961.

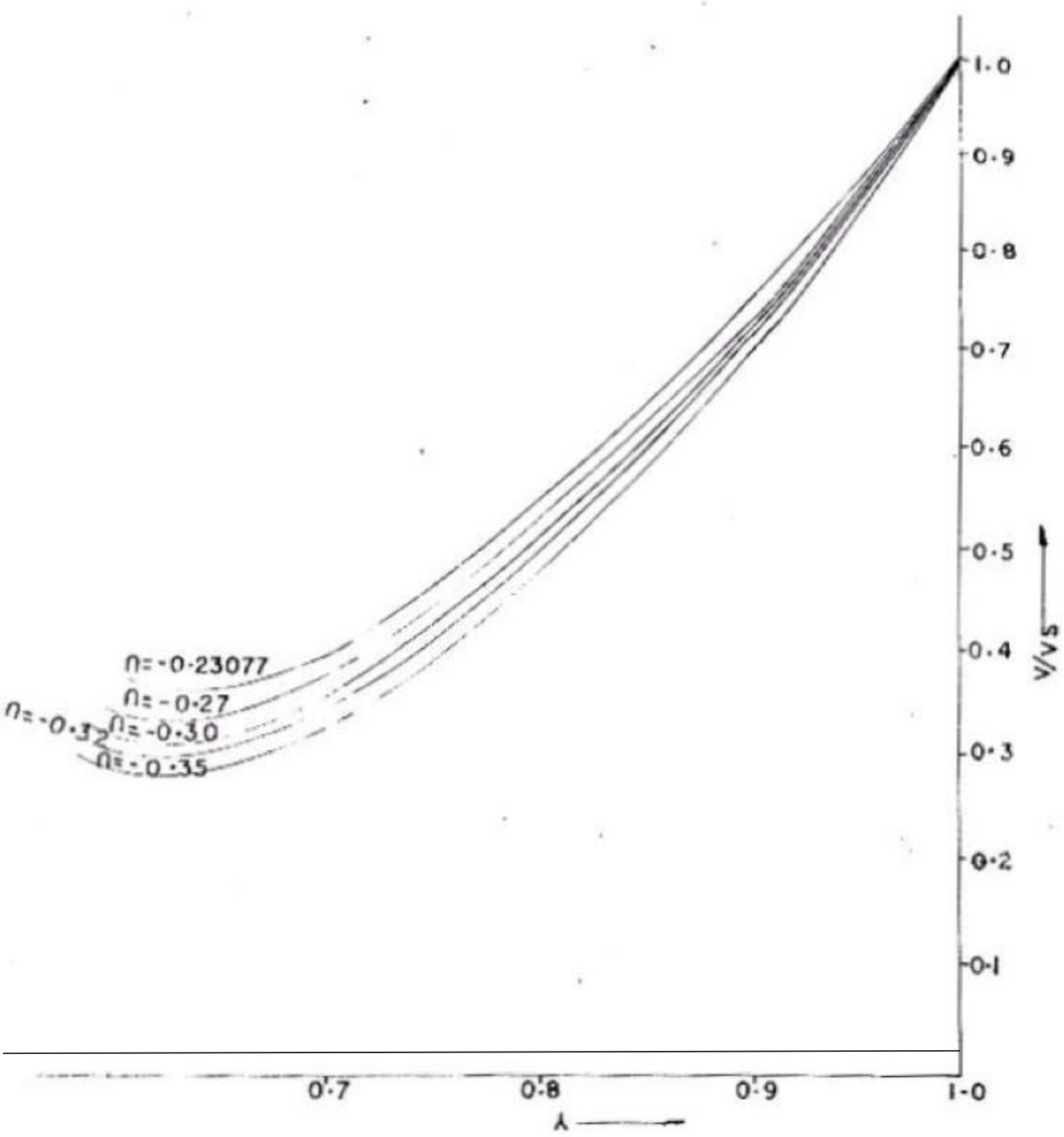

Fig. 1: Velocity Distribution for Spherical Symmetry 


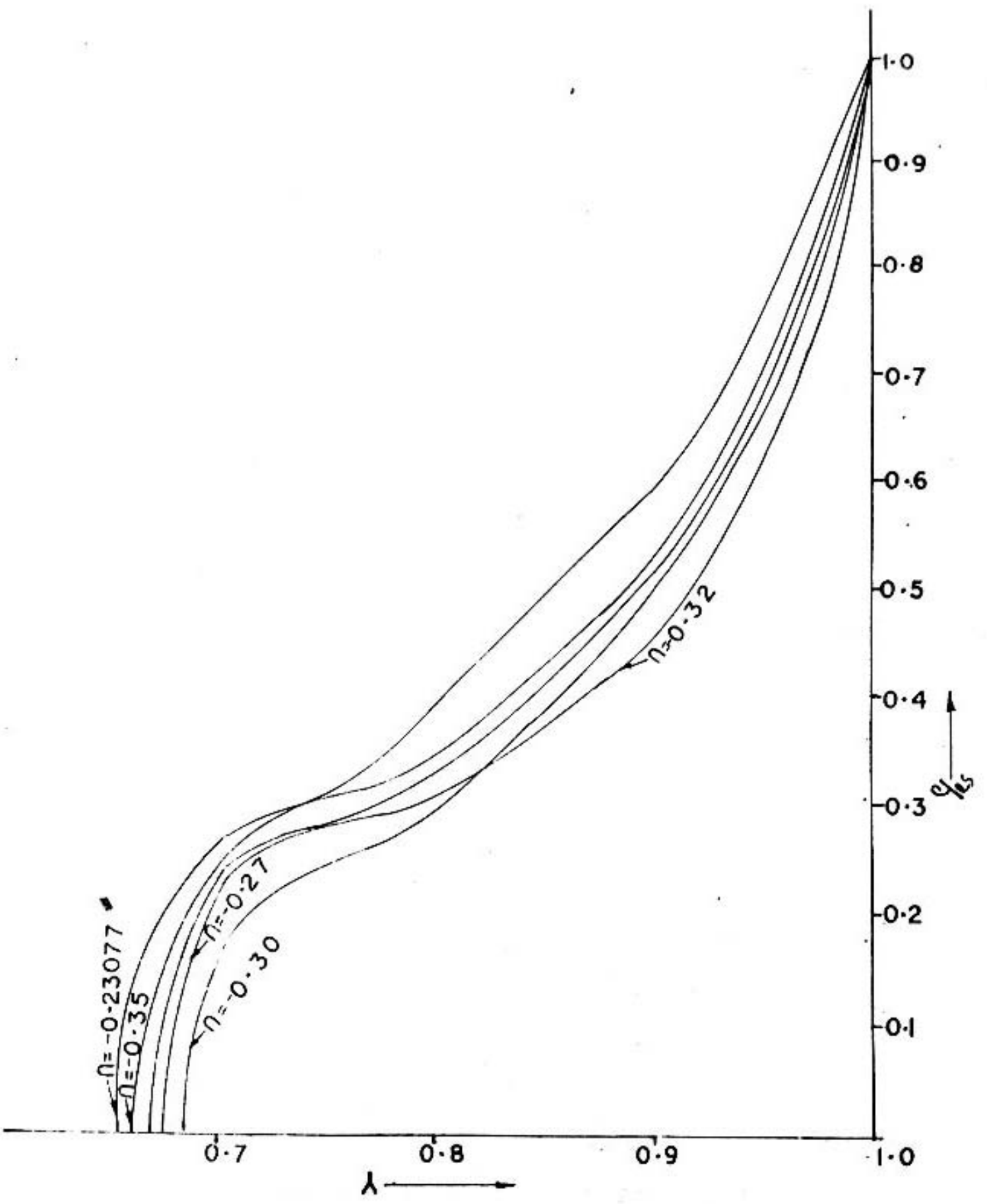

Fig. 2: Density Distribution For Spherical Symmetry 


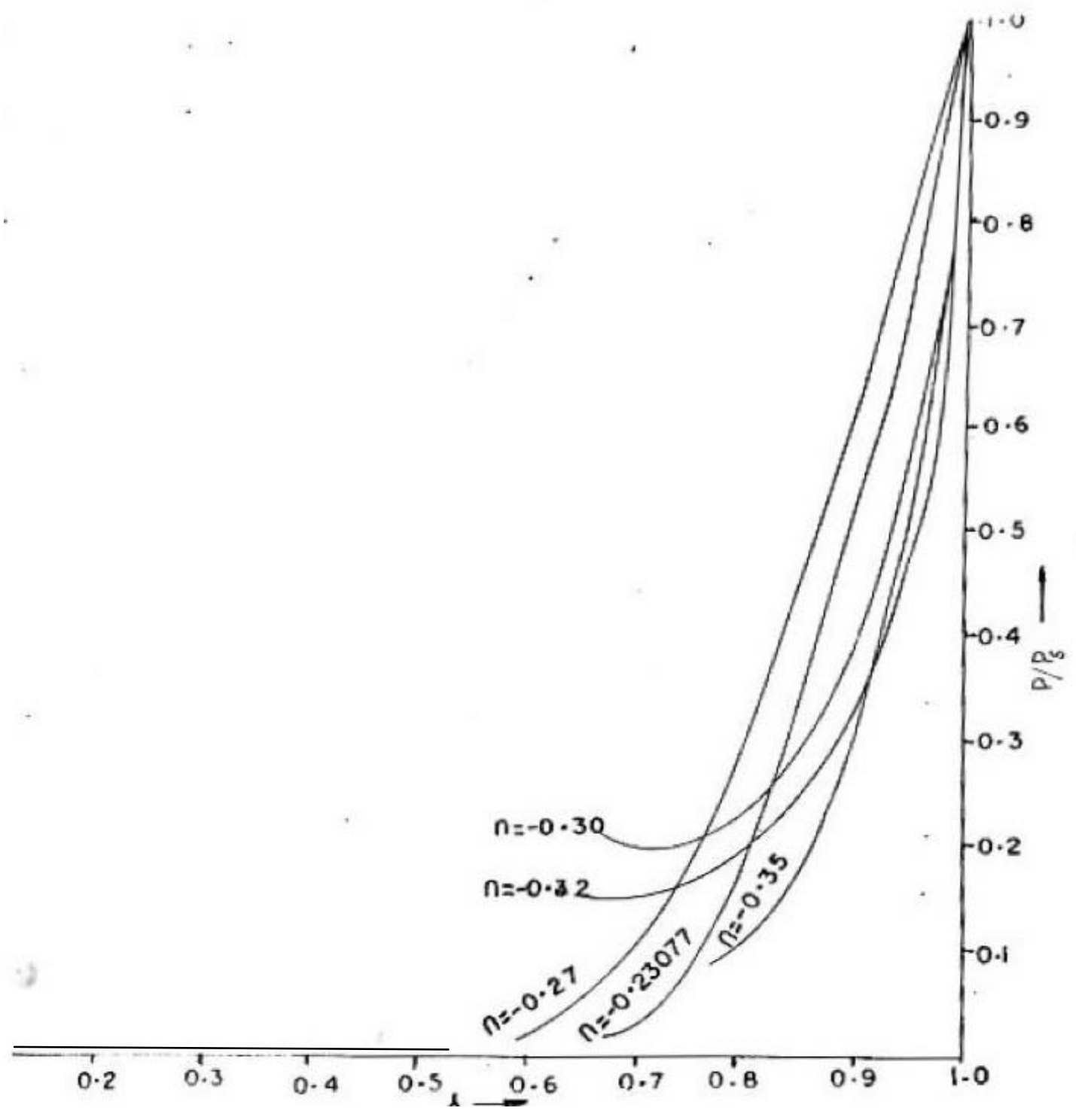

Fig. 3: Pressure Distribution For Spherical Symmetry 


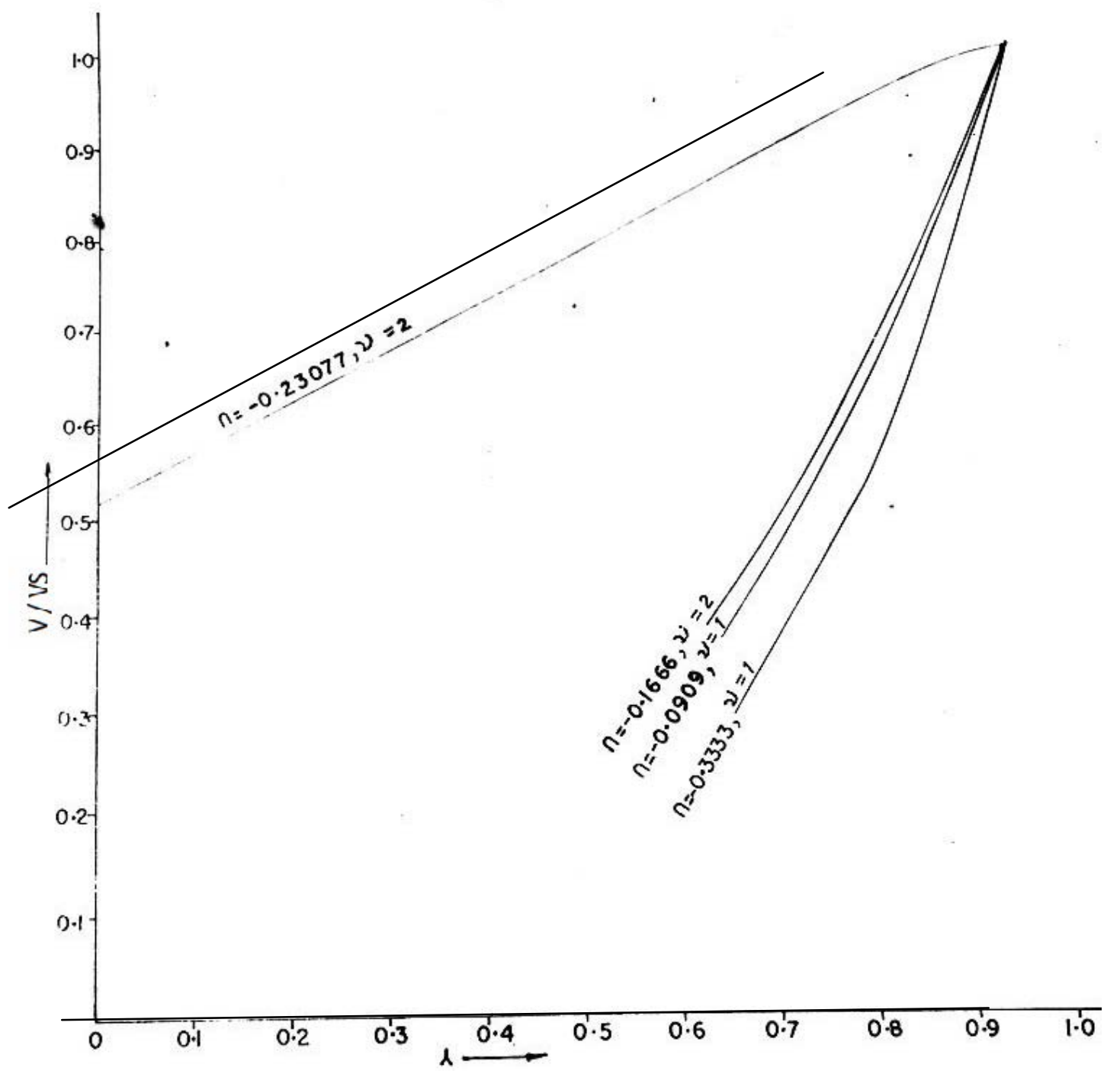

Fig. 4: Velocity Distribution Behind the Shock for Cases with Clyindrical and Plane Symmetry 


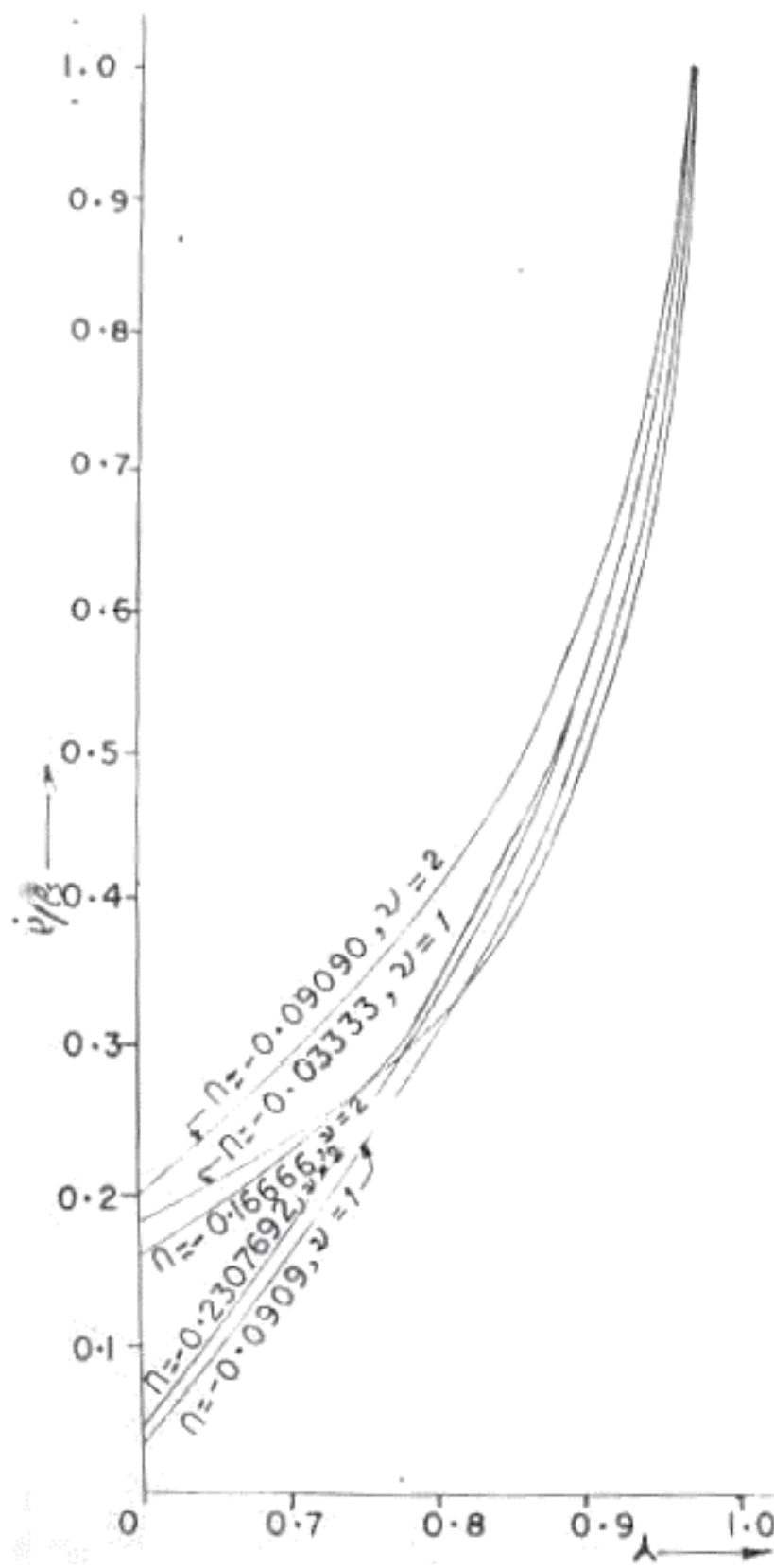

Fig. 5: Density Distribution Behind the Shock for Cases with Clyindrical and Plane Symmetry 


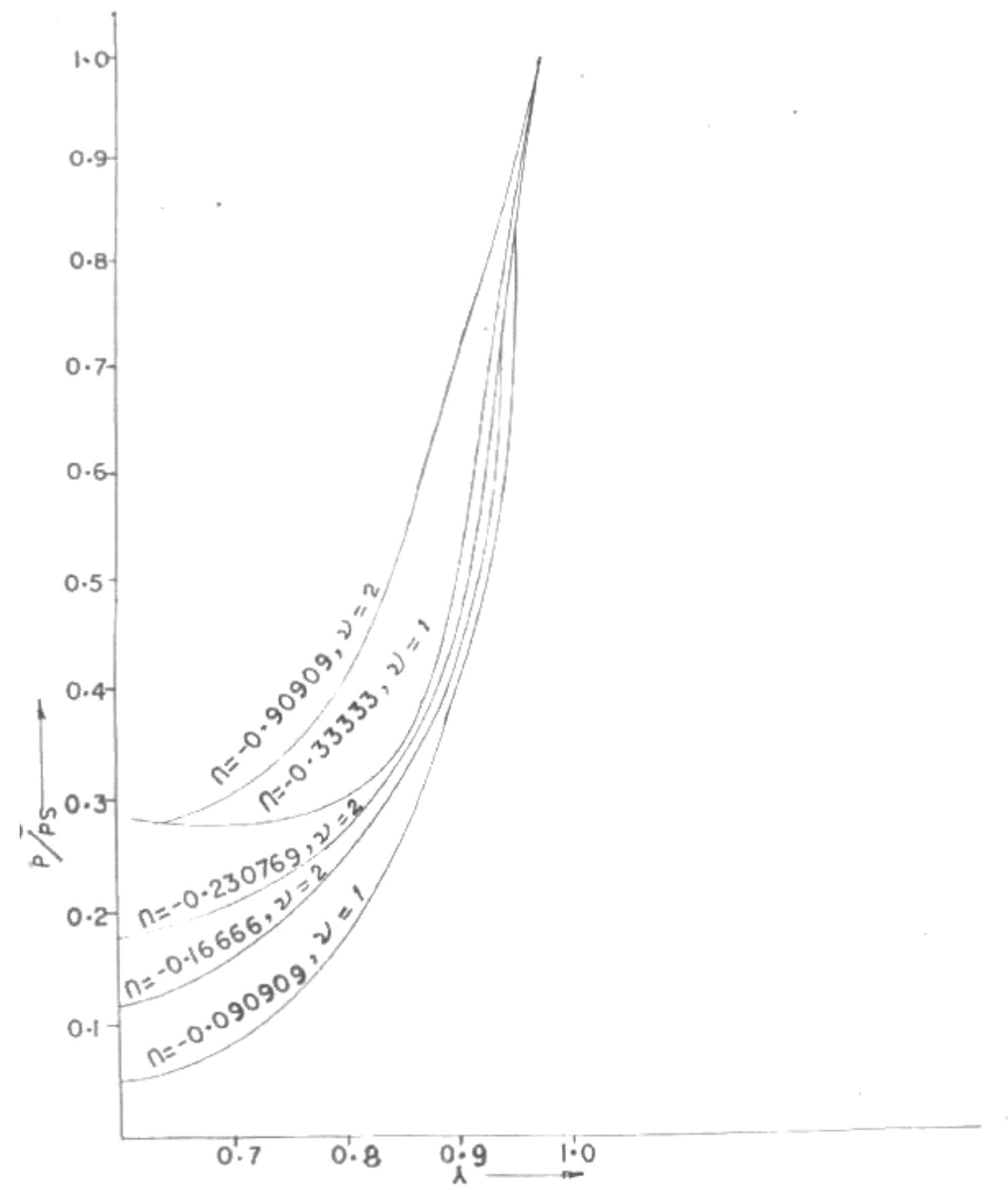

Fig. 6: Pressure Distribution Behind the Shock for Cases with Clyindrical and Plane Symmetry 